\title{
A Spacetime Cloak, or a History Editor
}

\author{
Martin W. McCall,* Alberto Favaro, and Paul Kinsler \\ Blackett Laboratory, Imperial College, Prince Consort Road, \\ London SW7 2AZ, United Kingdom.
}

\author{
Allan Boardman \\ Photonics and Nonlinear Science Group, \\ Joule Laboratory, Department of Physics, \\ University of Salford, Salford M5 4WT, United Kingdom.
}

(Dated: Wednesday $12^{\text {th }}$ December, 2012)

\begin{abstract}
We introduce a recipe for producing a new type of electromagnetic cloak, the electromagnetic spacetime cloak, which conceals events rather than mere objects. Non-emitting events occurring during a restricted period are never suspected by a distant observer. The cloak works by locally manipulating the speed of light of an initially uniform light distribution, whilst the light rays themselves always follow straight paths. Any 'perfect' spacetime cloak would necessarily rely upon the technology of electromagnetic metamaterials, which has already been shown to be capable of deforming light in ways hitherto unforseen - to produce for example an electromagnetic object cloak. Nevertheless, we show how it is possible to use intensity dependent refractive indices to construct an approximate STC, an implementation that would enable the distinct signature of successful event cloaking to be observed. Potential demonstrations include systems that apparently violate quantum statistics, 'interrupt-without-interrupt' computation on convergent data channels, and the illusion of a Star-Trek transporter.
\end{abstract}

\footnotetext{
*Electronic address: m.mccall@imperial.ac.uk
} 


\section{INTRODUCTION}

Wouldn't it be amazing if, in an age of ubiquitous surveillance, a method could be devised to somehow remove a piece of the history that would otherwise be recorded by a surveying camera? A safe-cracker would be able, for a brief time, to enter a scene, open the safe, remove its contents, close the door and exit the scene, whilst the record of a surveillance camera apparently showed that the safe door was closed all the time. Although this sounds like science fiction, the lesson from metamaterials research in the last decade has taught us that within certain restrictions such speculations are not fantasy. We here show how the magic of editing history can be achieved by introducing the concept of the space-time cloak (STC). The proposal opens up a new paradigm for electromagnetic cloaking and has major implications for research in metamaterials, slow light, and phase modulation. Metamaterials already bend light in unconventional ways, producing aberration-free lenses [1], or spatial 'object' cloaks which coax electromagnetic radiation around a spatial void invisible to outside observers [2-4]. These latter developments are extensions of our historical and pre-historical abilities - that light can bend whilst traversing transparent materials has been known at least since humans went fishing with spears; even the controlled bending of light in prisms and lenses has been known for centuries. In contrast, the STC utilises time-dependent nanometer engineered electromagnetic metamaterials to create a temporal void within which events can be hidden. Remarkably, our spacetime 'event' cloak leaves light rays undeviated from their expected linear spatial trajectories, and instead works by dividing illuminating light into a leading part which is speeded up and passes before a collection of events occur, and a trailing part which is slowed down and passes after they have occurred. This process is thus utterly distinct from a spatial "object" cloak, which instead achieves concealment by bending light around an object. The STC opens a temporary corridor through which energy, information, and matter can be manipulated or transported undetected. Once the concealed passage has been used, the STC closes by slowing the leading part of the light, whilst speeding up the trailing part, leaving no trace of the cloak, or event, on the field profile. To a distant observer, any non-emitting object whose presence persisted throughout the lifetime of the STC will have had a finite interval excised from its history. Any object which emits light during STC operation will have that part of its history temporally compressed so that if not absorbed, it will appear to the observer as if occurring in a single instant. We here outline the theory and 
design of the spacetime cloak and describe potential applications: hiding events from some observers but not others, using the 'time-gap' to generate apparent violations of quantum statistics, allowing the covert interruption of non-interruptible computation and/or signal processing operations, and creating the illusion of a Star-Trek transporter.

\section{THE SPACETIME CLOAK CONCEPT}

Our method for redacting history is based on applying the principles of transformation optics to produce an electromagnetic cloak working in both space and time, rather than in just space. Transformation optics relies on mimicking a coordinate transformation applied to Maxwell's vacuum equations by means of an inhomogeneous dielectric/magnetic medium. A coordinate transformation $\mathbf{r} \rightarrow \mathbf{r}^{\prime}$, taking straight lines to curves, transforms the electromagnetic field so that in the new system

$$
\begin{array}{ll}
\nabla^{\prime} \times \mathbf{E}^{\prime}=-\frac{\partial \mathbf{B}^{\prime}}{\partial t}, & \nabla^{\prime} \cdot \mathbf{D}^{\prime}=0 \\
\nabla^{\prime} \times \mathbf{H}^{\prime}=\frac{\partial \mathbf{D}^{\prime}}{\partial t}, & \nabla^{\prime} \cdot \mathbf{B}^{\prime}=0 .
\end{array}
$$

Since the form of Maxwell's equations is preserved, the vacuum relations $\mathbf{D}=\epsilon_{0} \mathbf{E}, \mathbf{B}=\mu_{0} \mathbf{H}$ are replaced in the new coordinate system by appropriate inhomogeneous, anisotropic relations $\mathbf{D}^{\prime}=\boldsymbol{\epsilon}^{\prime}\left(\mathbf{r}^{\prime}\right) \cdot \mathbf{E}^{\prime}, \mathbf{B}^{\prime}=\boldsymbol{\mu}^{\prime}\left(\mathbf{r}^{\prime}\right) \cdot \mathbf{H}^{\prime}$. If instead of transforming coordinates, the original coordinate space $(\mathbf{r})$ is filled with a medium $\boldsymbol{\epsilon}(\mathbf{r}), \boldsymbol{\mu}(\mathbf{r})$, having the same inhomogeneous, anisotropic (and strictly speaking instantaneously responding) properties prescribed by $\boldsymbol{\epsilon}^{\prime}\left(\mathbf{r}^{\prime}\right), \boldsymbol{\mu}^{\prime}\left(\mathbf{r}^{\prime}\right)$, then the linear light paths of vacuum become curved, just as straight lines become curved under $\mathbf{r} \rightarrow \mathbf{r}^{\prime}$. Figure 1(a) shows how a spatial electromagnetic cloak [2] is produced via a time invariant planar transformation $(x, y) \rightarrow\left(x^{\prime}, y^{\prime}\right)$ that diverts light rays so as to create a void near the origin. The polar transformation $r^{\prime}=(1-a / b) r+a, \theta^{\prime}=\theta$, $z^{\prime}=z$ distorts linear rays travelling in vacuum from left to right to those shown. Equivalently, rays in an anisotropic dielectric with polar parameters $(\epsilon, \mu)_{r}=(1-a / r)\left(\epsilon_{r}, \mu_{r}\right)_{0}$, $(\epsilon, \mu)_{\theta}=(1-a / r)^{-1}\left(\epsilon_{\theta}, \mu_{\theta}\right)_{0},(\epsilon, \mu)_{z}=(1-a / b)^{-2}(1-a / r)\left(\epsilon_{z}, \mu_{z}\right)_{0}$ are curved in exactly the same way, to produce a cloak around a cylindrical object of radius $a$, centered at the origin [4]. 
For our spacetime cloak we consider space-time transformations $(x, t) \rightarrow\left(x^{\prime}, t^{\prime}\right)$ and restrict to light propagating forwards along the $x$-axis. In Fig. 1(b) for example, a spacetime transformation is carried out in the $(x, t)$ plane that is analogous to the spatial transformation carried out in the $(x, y)$ plane of Fig. 1(a). In contrast to the spatial cloak, where the direction of propagation in the $x-y$ plane is arbitrary, the vacuum light rays must follow the straight lines $x=c t+$ const. These rays are then mapped under the transformation to the curved rays shown so that the events within the disc surrounding the origin are avoided by the new rays. The new light trajectories are actualized when they propagate through a suitable inhomogeneous time-dependent medium, and will then curve around the event occurring at $x=0, t=0$. As discussed in the Appendix, the form of Maxwell's equations (Eqs. (1)-(2), but now with the addition that $\left.t \rightarrow t^{\prime}\right)$ is again preserved provided the equivalent medium is magneto-electric with the transverse field components obeying

$$
\begin{aligned}
& \left(\begin{array}{c}
D_{y} \\
D_{z}
\end{array}\right)=\epsilon(x, t)\left(\begin{array}{c}
E_{y} \\
E_{z}
\end{array}\right)+\beta(x, t)\left(\begin{array}{c}
H_{z} \\
-H_{y}
\end{array}\right) \\
& \left(\begin{array}{c}
B_{y} \\
B_{z}
\end{array}\right)=\beta(x, t)\left(\begin{array}{c}
-E_{z} \\
E_{y}
\end{array}\right)+\mu(x, t)\left(\begin{array}{c}
H_{y} \\
H_{z}
\end{array}\right) .
\end{aligned}
$$

The medium is thus in general required to be magneto-electric. In contrast to the spatial cloak, where light curves in the $(x, y)$ plane, 'curving' light in the $(x, t)$ plane now refers to different parts of the light paths speeding up and slowing down. The light proceeds only along the $x$-axis and does not curve in space. As a consequence, events in the neighborhood of the event at $(x, t)=(0,0)$ are never suspected by an observer located sufficiently far to the right. In fact, all events in the $y-z$ plane that occur near $(x, t)=(0,0)$ are similarly undetected. The concealment results from various parts of the light distribution slowing down and speeding up so as to avoid events near the spacetime origin. The intensity along the $x$-axis for different times is shown on the right of Fig. 1(b), indicating the formation of the intensity null that is fully developed at $t=0$. For later times the null closes up restoring a uniform intensity distribution so that the observer at $x=a$ records a constant intensity for all time. Since the events near $(x=0, t=0)$ are never illuminated, the observer to the right is unaware of their existence. In fact, all events in the $(y, z)$ plane that occur near $(x=0, t=0)$ are concealed, so that effectively a space-time corridor is opened along which 
non-radiating events, such as the movement of matter, exchange of information etc. can occur undetected.

The space-time cloak of Fig. 1(b) is symmetric, in that uniform illumination from the right will avoid the same cloaked events for an observer located sufficiently far to the left. As conceived with the symmetric transformation in the $x-t$ plane of Fig. 1(b), some rays in the medium will be required to have a phase speed exceeding the vacuum speed of light (e.g. ray $\mathrm{A}$ in the inset), and some rays very close to the cloaked region (e.g. ray B) propagate backwards in time. Super-luminal ray trajectories, which occur in purely spatial cloaks and in plasma propagation below the plasma frequency, are characterized by the phase velocity exceeding the vacuum speed of light, and are known to be compatible with special relativity [5]. Waves travelling backwards in time are precisely the interpretation of waves undergoing negative phase velocity propagation, or negative refraction, where the reversal of time in the wave's phase $\phi=\mathbf{k} \cdot \mathbf{r}-\omega t$ is equivalent to the reversal of the direction of the wave vector $\mathbf{k}$ with respect to electromagnetic flux [6]. However, both super-luminal and negative phase waves, though accessible through appropriate metamaterial design, can, through similar design ingenuity, both be avoided in the spacetime cloak, as we discuss next.

\section{SIMPLIFIED DESIGNS}

Referring to Figure 2, the coordinate transformation is now carried out against a background of a uniform medium of refractive index $n>1$, rather than vacuum. The complete transformation consists of a Lorentz transformation into a frame in which the rays are vertical, opening the void via a 'curtain map', followed finally by an inverse Lorentz transformation back into the medium rest frame (see Appendix for further details). The construction is such that all rays in Fig. 2(a) travel slower than the vacuum speed of light. The plots of Fig. 2(b) show a detailed calculation of the electromagnetic energy density in the equivalent medium defined by the transformation, where the hard edges of the map have been mollified so that the medium is not switched instantaneously. The energy null which develops as the cloak is switched on becomes zero at events near the space-time origin. Unlike the cloak of Fig. 1(b), this space-time cloak only works for light travelling in the $+x$ direction. An observer to the left of the origin does see the cloaked events, though time separations between the cloaked events are first speeded up, then time shifted, and then finally slowed 
down, before progression returns to normal.

The magneto-electric parameter, $\beta(x, t)$, in Eqs. (3)-(4) arises almost invariably whenever the transformation $(x, t) \rightarrow\left(x^{\prime}, t^{\prime}\right)$ mixes space and time. This occurs, for example, in a medium of refractive index $n$ moving with velocity $v$ wherein the constitutive relations revert to the Minkowski form [7], the magneto-electric parameter being then given by

$$
\beta(x, t)=\frac{v(x, t)}{c}\left[\frac{n^{2}-1}{1-n^{2} v^{2}(x, t) / c^{2}}\right] .
$$

The spacetime cloak therefore involves the construction of a metamaterial that mimics propagation in a medium with a velocity $v(x, t)$. The occurrence of the null can then be understood via the velocity addition formula $v^{\prime}(x, t)=[v(x, t)+c / n] /[1+v(x, t) /(c n)]$ where $v^{\prime}(x, t)$ is the velocity of light in the moving medium. Near $t=0$, the effective medium is arranged so that $v(x, t)$ is negative for $x<0$ and positive for $x>0$. The trailing and leading parts of the light are thus respectively slowed down and speeded up to produce the required intermediate null. Non-reciprocal bi-anisotropic metamaterials that mimic the constitutive relations for a moving medium have been studied by Tretyakov [8] who has shown that such media can in principle be constructed from small magnetized ferrite spheres combined with planar-chiral metallic inclusions [9].

A space-time cloak with a transformation of the form $t^{\prime}=t, x^{\prime}=x^{\prime}(x, t)$ is illustrated in Fig. 3 which only requires manipulation of $\epsilon(x, t)$ and $\mu(x, t)$. The transformation is devised such that the spatio-temporal variation of these parameters matches, so that the impedance is uniform. The refractive index, however, is switched from high to low before the central region, and from low to high after the central region. The price of this simplified design is that the index changes persist in the switched regions, so that unlike the previous designs, the medium surrounding the spatial origin is not returned to its original state after the cloak has operated. Periodic switching, as shown in Fig. 3, returns the medium to its original state every other cycle and, moreover, permits bi-directionality by alternating the forward cloak with an identical one for light travelling in the $-x$ direction. If the index is switched without any impedance matching (as was done for the first demonstrations of the spatial cloak [4]), then reflections will occur at the interfaces. The observer to the right would consequently notice abrupt changes of the overall field intensity, but still remain ignorant of the events occurring within the cloaked period. 


\section{DEMONSTRATIONS}

What effects might be observable using the space-time cloak? Fig. 4 shows a collage of possibilities based around what happens in the $y-z$ plane in the region near $x=0$ where/when the cloak operates. Filling this region with excited atoms (Fig. 4(a)) will cause the standard exponential fluorescence decay to be modified by the cloak, as emissions within the cloaked period will all appear to happen simultaneously to the distant observer to the right. The observed spike could be a useful experimental signature, since the required cloaking period need only be comparable to the spontaneous lifetime $(\sim \mathrm{ns})$, corresponding to a dimension of $\sim 30 \mathrm{~cm}$ for the cloaked central region. Measurements of the coherence and statistics of light signals emitted by such a cloaked source will also yield anomalous results, even those of a quantum origin such as photon bunching or anti-bunching. Fig. 4(b) proposes a specific application in signal processing where two channels, proceeding along the $x$ and $y$-directions respectively, cross at $x=0$. Through the operation of the space-time cloak occasional signals along the $y$ channel can be processed as a priority, whilst processing and computation proceeds seamlessly, without interruption along the $x$ channel, achieving effectively an 'interrupt-without-interrupt'. Finally, the space-time cloak can achieve the illusion of a matter transporter (Fig.4(c)), in which an object moves from $\left(y_{1}, z_{1}\right)$ to $\left(y_{2}, z_{2}\right)$ during the cloak's operation. The observer to the right sees the object disappear at $\left(y_{1}, z_{1}\right)$ to then instantaneously reappear at $\left(y_{2}, z_{2}\right)$.

It is emphasised that all the above demonstrations only require light propagation in one dimension since producing a temporary null in a collimated wave is all that is necessary to conceal events near $x=0$ in the $y-z$ plane for a limited period. However, it is clear that the spacetime cloak concept can be extended to more spatial dimensions where the illuminating light is not collimated. A spherical wave from a point source, for example, illuminates a set of events lying on a sphere at radius $R$ from the origin. A spacetime cloak concealing these events will do so for distant observers positioned at any viewing angle.

\section{IMPLEMENTATION}

How practical is it to build a spacetime cloak? As with the original proposals for a spatial cloak, we have neglected dispersion whose effect in this case will be to constrain the rate at 
which the cloak can be opened and closed. Fig. 5 shows a scheme to implement the design of Fig. 3 using nonlinear optical fibre. The cloak is opened in fibre A, is held open in fibre B (which contains the core cloak region), and is closed again in fibre C. The primary operation is achieved by means of two control fields whose intensity allows the refractive index in A (C) to be modulated from low (high) to high (low). This is achieved using the nonlinear refractive index properties of the fibre (e.g. silica). The illumination field is affected by the refractive index shifts (i.e. by the cloak), but should have an intensity modulation far weaker than that used by the control fields. The cloak opens up a space-time gap in the intensity profile of the illumination field. This gap could be detected by monitoring leaky spot(s) on fibre B, and looking for the intensity $\operatorname{dip}(\mathrm{s})$ in the illumination. Alternatively, a signal field could be injected directly into fibre B, and would therefore always be present, i.e. before, during, and after the cloaking from the illumination field. By monitoring the signal as it is coupled out, the spacetime modulation on it from the illumination and the effect of nonlinear refractive index could be measured. For a brief period, the presence of the signal field will be undetectable to the surveiling illumination field and changes to the signal field during the cloaking period would leave no signature on the illumination field. The group velocity of the on/off transitions must be no slower than the slow phase velocity, and no faster than the fast phase velocity. This ensures that the control/cloaking index transitions always remains inside the cloak. Good dispersion control would be required (probably using photonic crystal fibre). If the various fields all have distinct frequencies then they can be coupled in and out efficiently and distinctly. The fibre parameters illustrated in Fig. 5 are optimistic but accessible (though harmonic generation would also need to be considered). Similarly, all coupling is assumed to be perfect, although slight leakage of control field A into fibre $\mathrm{B}$ and $\mathrm{C}$ should be tolerable.

\section{CONCLUSION}

We have introduced a fundamentally new concept of electromagnetic cloaking that exploits the transformation optics algorithm in one space and one time dimension. The result is a cloak that operates in a fundamentally distinct way to the purely spatial, or object cloak, in that it conceals events by curving light rays in spacetime rather than space. Although this sets new challenges for metamaterial design, we have shown how these challenges can 
be minimized through judicious manipulation of the refractive index of the meta-medium. We have proposed a proof-of-principle design based on nonlinear fiber optics that could demonstrate the signature of spacetime cloaking, and how the concept could find practical application in signal processing.

We are sure that there are many other possibilities that are opened up by our introduction of the concept of the space-time cloak.

Whilst the camera is said to never lie, it doesn't always tell the whole truth.

\section{APPENDIX}

\section{A. Calculating the material parameters for the spacetime cloak}

Calculating the required spatial and temporal material properties of the space-time cloak, and the resultant distribution of electromagnetic energy is most easily carried out using a covariant approach. We take Cartesian-Lorentz coordinates indexed as $\alpha=0,1,2,3$, i.e. $\left\{x^{\alpha}\right\}=(c t, x, y, z)$. The source-free Maxwell equations in a linear medium responding instantaneously to electromagnetic excitation may be expressed as

$$
\frac{\partial}{\partial x^{\beta}}\left(\chi^{\alpha \beta \mu \nu} F_{\mu \nu}\right)=0
$$

where $F_{\mu \nu}$ is the electromagnetic field tensor given by (in units where $c=1$ )

$$
F_{\mu \nu}=\left(\begin{array}{cccc}
0 & -E_{x} & -E_{y} & -E_{z} \\
E_{x} & 0 & B_{z} & -B_{y} \\
E_{y} & -B_{z} & 0 & B_{x} \\
E_{z} & B_{y} & -B_{x} & 0
\end{array}\right)
$$

The fourth rank object $\chi^{\alpha \beta \mu \nu}$ relates $F_{\mu \nu}$ to $G^{\alpha \beta}$ according to

$$
G^{\alpha \beta}=\chi^{\alpha \beta \mu \nu} F_{\mu \nu}
$$

where

$$
G^{\alpha \beta}=\left(\begin{array}{cccc}
0 & D_{x} & D_{y} & D z \\
-D_{x} & 0 & H_{z} & -H_{y} \\
-D_{y} & -H_{z} & 0 & H_{x} \\
-D_{z} & H_{y} & -H_{x} & 0
\end{array}\right)
$$


i.e. $G^{\alpha \beta}$ contains the fields $\mathbf{D}$ and $\mathbf{H}$. The fourth rank object $\chi^{\alpha \beta \mu \nu}$, contains the electromagnetic material parameters. In non-covariant notation we have

$$
\left(\begin{array}{l}
\mathrm{D} \\
\mathbf{H}
\end{array}\right)=\left(\begin{array}{cc}
-\boldsymbol{\epsilon} & \boldsymbol{\alpha} \\
-\boldsymbol{\alpha}^{T} & \boldsymbol{\mu}^{-1}
\end{array}\right)\left(\begin{array}{c}
-\mathbf{E} \\
\mathrm{B}
\end{array}\right)
$$

where $T$ indicates transpose. In fact, $\chi^{\alpha \beta \mu \nu}$ is a fourth rank tensor density and transforms according to $[10]$

$$
\chi^{\alpha^{\prime} \beta^{\prime} \mu^{\prime} \nu^{\prime}}=\left|\operatorname{det}\left(L_{\lambda}^{\lambda^{\prime}}\right)\right|^{-1} L_{\alpha}^{\alpha^{\prime}} L_{\beta}^{\beta^{\prime}} L_{\mu}^{\mu^{\prime}} L_{\nu}^{\nu^{\prime}} \chi^{\alpha \beta \mu \nu}
$$

where $L_{\alpha}^{\alpha^{\prime}}=\partial x^{\alpha^{\prime}} / \partial x^{\alpha}$.

For a transformation restricted to the $\left(x^{0}, x^{1}\right)$ plane and a base constitutive tensor for which the non-zero elements are $\chi^{0101}=\chi^{0202}=\chi^{0303}=-\epsilon ; \chi^{2323}=\chi^{3131}=\chi^{1212}=\mu^{-1}$ we find the non-zero elements of $\chi^{\alpha^{\prime} \beta^{\prime} \mu^{\prime} \nu^{\prime}}$ to be

$$
\begin{aligned}
\chi^{0^{\prime} 1^{\prime} 0^{\prime} 1^{\prime}}=\left|L_{\alpha}^{\alpha^{\prime}}\right|^{-1}\left(L_{0}^{0^{\prime}} L_{1}^{1^{\prime}} L_{0}^{0^{\prime}} L_{1}^{1^{\prime}}-L_{0}^{0^{\prime}} L_{1}^{1^{\prime}} L_{1}^{0^{\prime}} L_{0}^{1^{\prime}}\right. \\
\left.\quad+L_{1}^{0^{\prime}} L_{0}^{1^{\prime}} L_{1}^{0^{\prime}} L_{0}^{1^{\prime}}-L_{1}^{0^{\prime}} L_{0}^{1^{\prime}} L_{0}^{0^{\prime}} L_{1}^{1^{\prime}}\right)(-\epsilon) \equiv-\epsilon_{\|} \\
\chi^{0^{\prime} 2^{\prime} 0^{\prime} 2^{\prime}}=\left|L_{\alpha}^{\alpha^{\prime}}\right|^{-1}\left(-\epsilon L_{0}^{0^{\prime}} L_{0}^{0^{\prime}}+\mu^{-1} L_{1}^{0^{\prime}} L_{1}^{0^{\prime}}\right)=\chi^{0^{\prime} 3^{\prime} 0^{\prime} 3^{\prime}} \equiv-\epsilon_{\perp} \\
\chi^{2^{\prime} 3^{\prime} 2^{\prime} 3^{\prime}}=\left|L_{\alpha}^{\alpha^{\prime}}\right|^{-1} \mu^{-1} \equiv \mu_{\|}^{-1} \\
\chi^{3^{\prime} 1^{\prime} 3^{\prime} 1^{\prime}}=\left|L_{\alpha}^{\alpha^{\prime}}\right|^{-1}\left(-\epsilon L_{0}^{1^{\prime}} L_{0}^{1^{\prime}}+\mu^{-1} L_{1}^{1^{\prime}} L_{1}^{1^{\prime}}\right)=\chi^{1^{\prime} 2^{\prime} 1^{\prime} 2^{\prime}} \equiv \mu_{\perp}^{-1} \\
\chi^{0^{\prime} 2^{\prime} 1^{\prime} 2^{\prime}}=\left|L_{\alpha}^{\alpha^{\prime}}\right|^{-1}\left(-\epsilon L_{0}^{0^{\prime}} L_{0}^{1^{\prime}}+\mu^{-1} L_{1}^{0^{\prime}} L_{1}^{1^{\prime}}\right)=-\chi^{0^{\prime} 3^{\prime} 3^{\prime} 1^{\prime}}=\chi^{1^{\prime} 2^{\prime} 0^{\prime} 2^{\prime}}=-\chi^{3^{\prime} 1^{\prime} 0^{\prime} 3^{\prime}} \equiv \alpha .
\end{aligned}
$$

The material relations (10) can thus be expressed alternatively as

$$
\begin{aligned}
D_{x} & =\epsilon_{\|} E_{x}, B_{x}=\mu_{\|} H_{x} \\
\left(\begin{array}{c}
D_{y} \\
D_{z}
\end{array}\right) & =\left(\epsilon_{\perp}+\alpha^{2} \mu_{\perp}\right)\left(\begin{array}{c}
E_{y} \\
E_{z}
\end{array}\right)+\alpha \mu_{\perp}\left(\begin{array}{c}
H_{z} \\
-H_{y}
\end{array}\right) \\
\left(\begin{array}{c}
B_{y} \\
B_{z}
\end{array}\right) & =\alpha \mu_{\perp}\left(\begin{array}{c}
-E_{z} \\
E_{y}
\end{array}\right)+\mu_{\perp}\left(\begin{array}{c}
H_{y} \\
H_{z}
\end{array}\right)
\end{aligned}
$$

Eqs. (18) and (19) are seen to be of the same form as the Eqs. 3 and 4. 


\section{B. The Curtain Map in Detail}

The curtain map of Fig. 2 consists of successive space-time coordinate transformations $(x, t) \rightarrow(\bar{x}, \bar{t}) \rightarrow\left(\bar{x}^{\prime}, \bar{t}^{\prime}\right) \rightarrow\left(x^{\prime}, t^{\prime}\right)$ as shown in Fig. 6. Light propagating in a uniform medium of refractive index $n$ is represented by the straight sub-luminal world lines of (a). The transition from (a) to (b) is a Lorentz boost with speed $v=c / n$ applied according to

$$
\begin{aligned}
\bar{x} & =\left(1-n^{-2}\right)^{-1 / 2}(x-c t / n), \\
\bar{t} & =\left(1-n^{-2}\right)^{-1 / 2}(t-x / n c) .
\end{aligned}
$$

This produces the vertical photon world lines of (b) wherein the rectangular box $(|c \bar{t}|<$ $n \sigma,|\bar{x}|<\sigma)$ is identified. Within this rectangle a 'curtain map' then operates as

$$
\begin{aligned}
& \bar{x}^{\prime}=\left(\frac{\delta+|c \bar{t}|}{\delta+n \sigma}\right)[\bar{x}-\operatorname{sgn}(\bar{x}) \sigma]+\operatorname{sgn}(\bar{x}) \sigma, \\
& \bar{t}^{\prime}=\bar{t}
\end{aligned}
$$

with points outside the rectangle being mapped to themselves. Shown is the curtain map for which $\sigma=1, n=2, \delta=0.5$. Inverse Lorentz transforming back to the medium rest frame, where the coordinates are now $\left(x^{\prime}, t^{\prime}\right)$, yields the obliquely opened curtain as shown in (d). The deformed photon world lines in (d) all have positive gradients (i.e. propagate forwards in time) and have speeds (determined by the gradient of the world line) $\leq c / n$. The hard edges of the curtain map induced by the use of $|\cdot|$ and $\operatorname{sgn}(\cdot)$ functions in Eq. (22) may be softened by the use of suitable mollifier functions as explained in the next Section.

\section{Mollifying the Hard Edges of the Curtain Map}

As defined above, the composite map $(x, t) \rightarrow(\bar{x}, \bar{t}) \rightarrow\left(\bar{x}^{\prime}, \bar{t}^{\prime}\right) \rightarrow\left(x^{\prime}, t^{\prime}\right)$ is 'hard-edged', meaning that sections of the intensity at a given $t^{\prime}$ will change abruptly from zero inside the void region, to a finite value outside. This would require unrealistic instantaneous changes in the material parameters at the edge of the cloak. In order to soften these edges we applied the following procedure.

In the vertical ray frame (i.e. Fig. 6(b)) the functions $\operatorname{sgn}(\xi)$ and $|\xi|$ in Eq. (22) are replaced $\tanh (\xi / \Delta)$ and $\xi \tanh (\xi / \Delta)$ respectively, where $\Delta$ is a small parameter. Note that 
as $\Delta \rightarrow 0, \tanh (\xi / \Delta) \rightarrow \operatorname{sgn}(\xi)$ and $\xi \tanh (\xi / \Delta) \rightarrow|\xi|$. For points inside the rectangle Eqs. (22) and (23) are therefore replaced with

$$
\begin{aligned}
& \bar{x}_{\text {in }}^{\prime}=\left[\left(\frac{\delta+c \bar{t} \tanh (c \bar{t} / \Delta)}{\delta+n \sigma}\right)[\bar{x}-\tanh (\bar{x} / \Delta) \sigma]+\tanh (\bar{x} / \Delta) \sigma\right], \\
& \bar{t}_{\text {in }}^{\prime}=\bar{t} .
\end{aligned}
$$

The mollified curtain map of Eqs. (24) and (25) is continuous and differentiable at the origin. Points outside the rectangle $(|\bar{x}|>\sigma,|c \bar{t}|>n \sigma)$ are left unchanged as before (i.e. $\bar{x}_{\text {out }}=\bar{x}, \bar{t}_{\text {out }}=\bar{t}$. . To avoid discontinuities at the boundary of the box, the maps inside the rectangle and outside the rectangle are graded into each other by means of the mollified rectangular 'top hat' function:

$$
\rho(\bar{x}, c \bar{t})=\frac{1}{4}\left[\tanh \left(\frac{c \bar{t}+n \sigma}{\Delta}\right)-\tanh \left(\frac{c \bar{t}-n \sigma}{\Delta}\right)\right]\left[\tanh \left(\frac{\bar{x}+\sigma}{\Delta}\right)-\tanh \left(\frac{\bar{x}-\sigma}{\Delta}\right)\right] .
$$

Note that as $\Delta \rightarrow 0$ Eq. (26) becomes a rectangular top-hat function. The maps inside and outside the rectangle are then graded into each other according to

$$
\begin{aligned}
\bar{x}^{\prime} & =\rho \bar{x}_{\text {in }}+(1-\rho) \bar{x}_{\text {out }}, \\
\bar{t}^{\prime} & =\bar{t} .
\end{aligned}
$$

This procedure ensures that the resultant map $(\bar{x}, \bar{t}) \rightarrow\left(\bar{x}^{\prime}, \bar{t}^{\prime}\right)$ defined by combining Eqs. (27) and (28) with Eqs. (24)-(26) is continuous at the boundary of the rectangle. The complete map $(x, t) \rightarrow\left(x^{\prime}, t^{\prime}\right)$ is then specified via the Lorentz transformation of Eqs. (20)-(21) sending $(x, t) \rightarrow(\bar{x}, \bar{t})$, the mollified curtain map of Eqs. (27)-(28) sending $(\bar{x}, \bar{t}) \rightarrow\left(\bar{x}^{\prime}, \bar{t}^{\prime}\right)$, followed by the inverse Lorentz transformation sending $\left(\bar{x}^{\prime}, \bar{t}^{\prime}\right) \rightarrow\left(x^{\prime}, t^{\prime}\right)$. The composite map $(x, t) \rightarrow\left(x^{\prime}, t^{\prime}\right)$, with $\Delta=0.08$, was used to generate Fig. 2 in the paper by calculating numerically the transformed material parameters using Eq. (12)-(16), and then calculating the electromagnetic energy density according to

$$
U=\frac{1}{2}(\mathbf{D} \cdot \mathbf{E}+\mathbf{B} \cdot \mathbf{H})
$$

Acknowledgements Support from EPSRC grant no. EP/E031463/1 and a Leverhulme 
Embedding Emerging Disciplines award is acknowledged.

[1] Pendry, J. B., Negative Refraction Makes a Perfect Lens, Phys. Rev. Lett. 85, 3966 (2000).

[2] Pendry, J.B., Schurig, D. \& Smith, D. R., Controlling Electromagnetic Fields, Science 312, 1780-1782 (2006).

[3] Leonhardt, U. \& Philbin, T.G. General Relativity in Electrical Engineering, New J. of Physics 8, 247 (2006).

[4] Schurig, D., Mock, J. J., Justice, B. J., Cummer, S. A., Pendry, J. B., Starr, A. F. \& Smith, D. R. Metamaterial Electromagnetic Cloak at Microwave Frequencies, Science 314, 977-980 (2006).

[5] Léon Brillouin, Wave Propagation and Group Velocity, First Ed., Academic Press, New York, 1960.

[6] Pendry, J.B., Time Reversal and Negative Refraction, Science 322, 71-73 (2009).

[7] McCall, M.W., Relativity and Mathematical Tools: Waves in Moving Media, American Journal of Physics 75, 1134-1140 (2007).

[8] Tretyakov, S.A., Nefedov, I.S. and Alitalo, P., Generalized Field-transforming Metamaterials, New Journal of Physics, 10, 115028 (2008).

[9] Tretyakov, S.A., On a Possibility to Imitate Media Moving with Superluminal Velocity, Third International Conference on Advanced Electromagnetic Materials in icrowves and Optics, London (2009).

[10] Post, E. J., Formal Structure of Electromagnetics Dover (1997). 
(a)
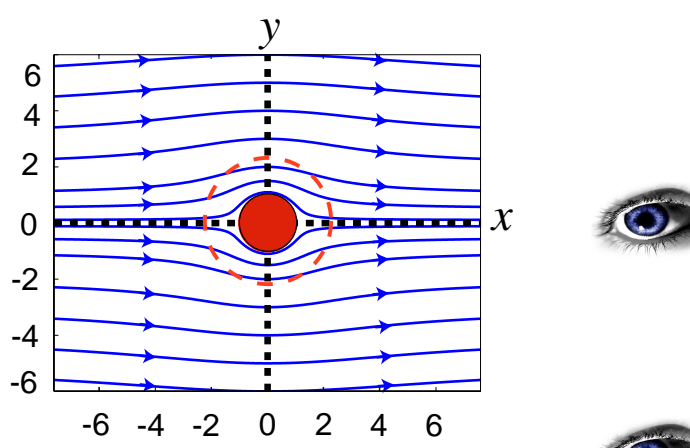

(b)

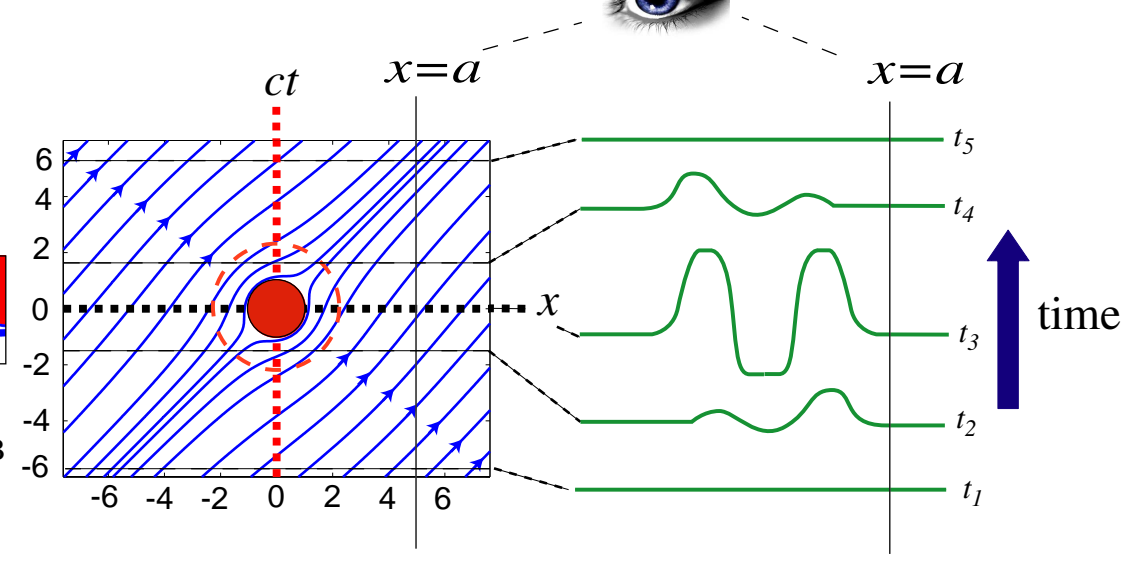

FIG. 1: (a) Conventional spatial cloak based on a planar transformation $(x, y) \rightarrow\left(x^{\prime}, y^{\prime}\right)$. An observer located to the far right on the $x$-axis does not see the object. (b) Spacetime cloak. An analogous coordinate transformation is used, but now in $(x, t)$ rather than $(x, y)$. The cloak now conceals events near the space-time origin. The intensity distribution for various times is shown on the right indicating the formation and subsequent evaporation of the intensity null that is fully developed at $t=0$. The observer to the right never suspects the occurrence of any non-radiating events near the space-time origin and sees a uniform intensity for all time. 

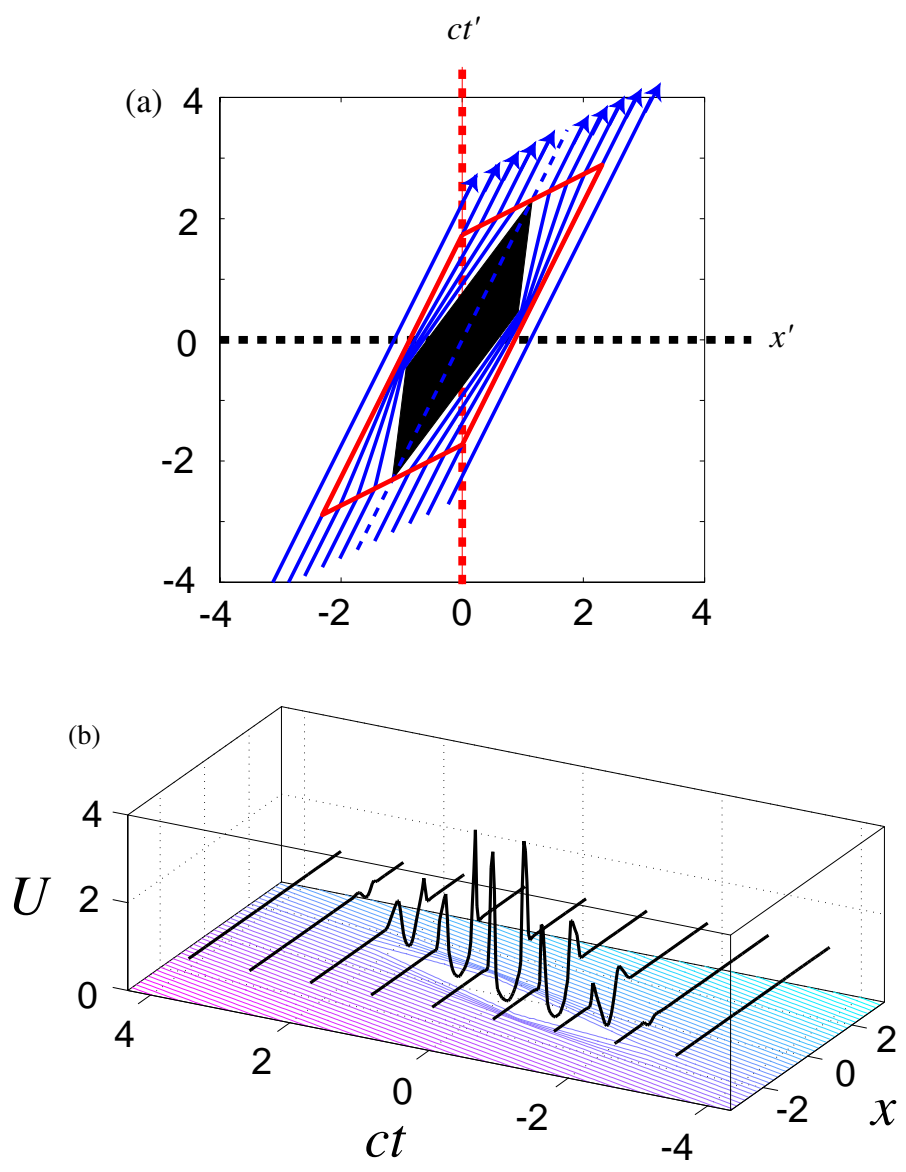

FIG. 2: (a) Construction of a sub-luminal space-time cloak. As with Fig. 1(b) the map $(x, t) \rightarrow$ $\left(x^{\prime} t^{\prime}\right)$ creates a void near the space-time origin. However, the base space is filled with a medium of refractive index $n$ rather than vacuum, so that prior to the transformation light rays are straight lines of gradient $n$. The transformation is a composition of a Lorentz boost, $(x, t) \rightarrow(\bar{x}, \bar{t})$, with velocity $v=c / n$, followed by applying a 'curtain map' $\bar{x}=\left[\left(\frac{\delta+|c \bar{t}|}{\delta+n \sigma}\right)(\bar{x}-\operatorname{sgn}(\bar{x}) \sigma)+\operatorname{sgn}(\bar{x}) \sigma\right], \bar{t}^{\prime}=$ $\bar{t}$, followed by an inverse Lorentz transformation, $\left(\bar{x}^{\prime}, \bar{t}^{\prime}\right) \rightarrow\left(x^{\prime}, t^{\prime}\right)$. (see Appendix for further details). Shown is the coordinate transformation $(x, t) \rightarrow\left(x^{\prime}, t^{\prime}\right)$ with $\sigma=1, n=2, \delta=0.5$ for the curtain map. The deformed rays all have positive gradients (i.e. propagate forwards in time) and have speed $\leq c$. The coordinate transformation $(x, t) \rightarrow\left(x^{\prime}, t^{\prime}\right)$ defines the spacetime transformation that yields Eqs. (1)-(2) with $t=t^{\prime}$. Alternatively, a material with the required $\epsilon(x, t), \mu(x, t), \beta(x, t)$, enables the space-time cloak to be realized in an actual medium. (b) Electromagnetic energy density for various times for the map of (a). The hard edges of the curtain map defined above have been softened by the use of mollifier functions (see Appendix), thus avoiding unrealistic instantaneous switching. The intensity is null at events near the spacetime origin. 


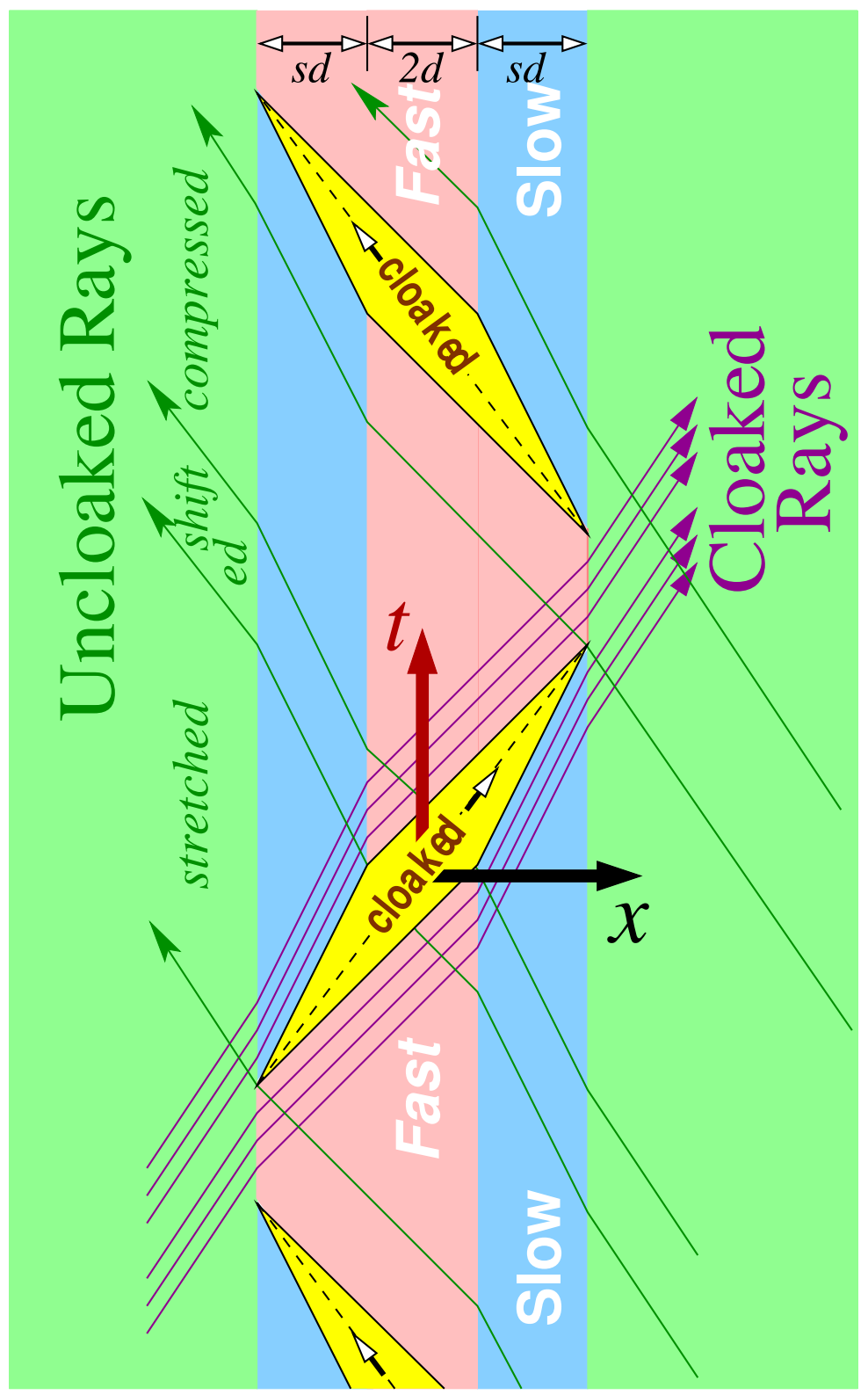

FIG. 3: Space-time cloak based on refractive index switching. (a) The base space is a medium of refractive index $n$ with an inner region of width $2 d$ surrounded by two outer regions of extent $s d$ identified. For $-(1+s) d \leq x<0$ the transformation is given by $x^{\prime}=(s+2)(s+1)^{-1} x+d$ for $t \leq n x$ and by $x^{\prime}=s(s+1)^{-1} x-d$ for $t>n x$. For $0 \leq x<(s+1) d$ the transformation is given by $x^{\prime}=s(s+1)^{-1} x+d$ for $t \leq n x$ and by $x^{\prime}=(s+2)(s+1)^{-1} x-d$ for $t>n x$. All other regions are unaffected. All rays are sub-luminal provided $n(1+1)(s+2)^{-1}<1$. Unlike the cloak of Fig. $2(a)$, a single cloaking operation does not return the system to its original state. Alternating cloak operations as shown permits periodic restoration of the medium to its original state by interleaving a cloak operating in the reverse direction. 


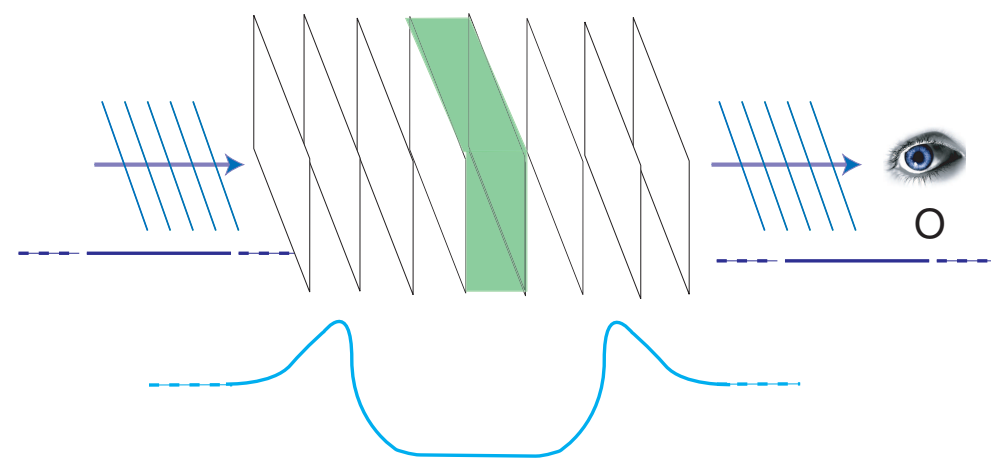

(a)
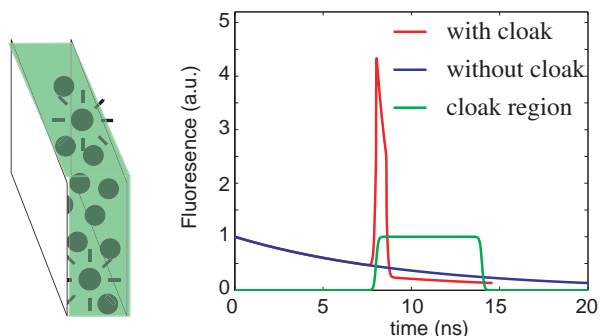

(c)

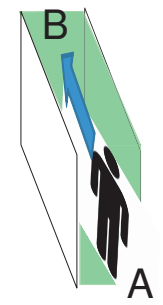

(b)

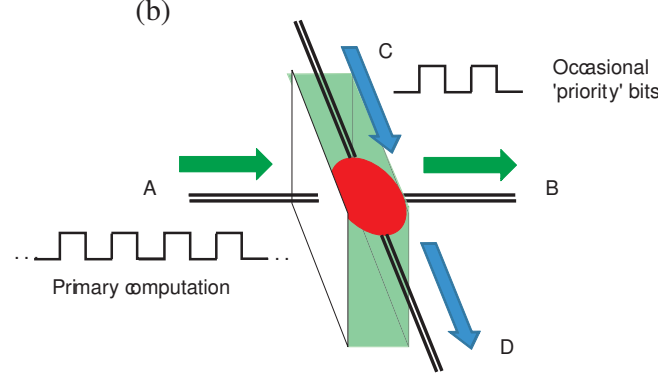

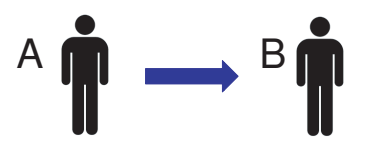

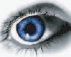

O

FIG. 4: Realization of the spacetime cloak as a set of addressable planes with metallic inclusions. Applications based on how the central region is filled as the intensity null passes over: (a) Excited atoms. The exponential fluorescence decay is observed with the emissions during cloak operation all apparently occurring simultaneously, resulting in an intensity spike, followed by resumption of the exponential decay. (b) 'Interrupt-without-interrupt'. An occasional signal (channel AB) and a primary computation (channel CD) converge at the same physical node. Suppose it is required to process the occasional signal, without interrupting the primary computation. The cloak operation opens a temporal window via which the occasional signal can be processed, whilst the primary computation is reconstituted after the cloak operation to appear as if uninterrupted. (c) A StarTrek transporter. Movement of non-radiating matter whilst the cloak is operating appears to the observer $\mathrm{O}$ to have moved from A to B instantaneously. 


\section{Basic optical-fibre implementation of a space-time cloak}

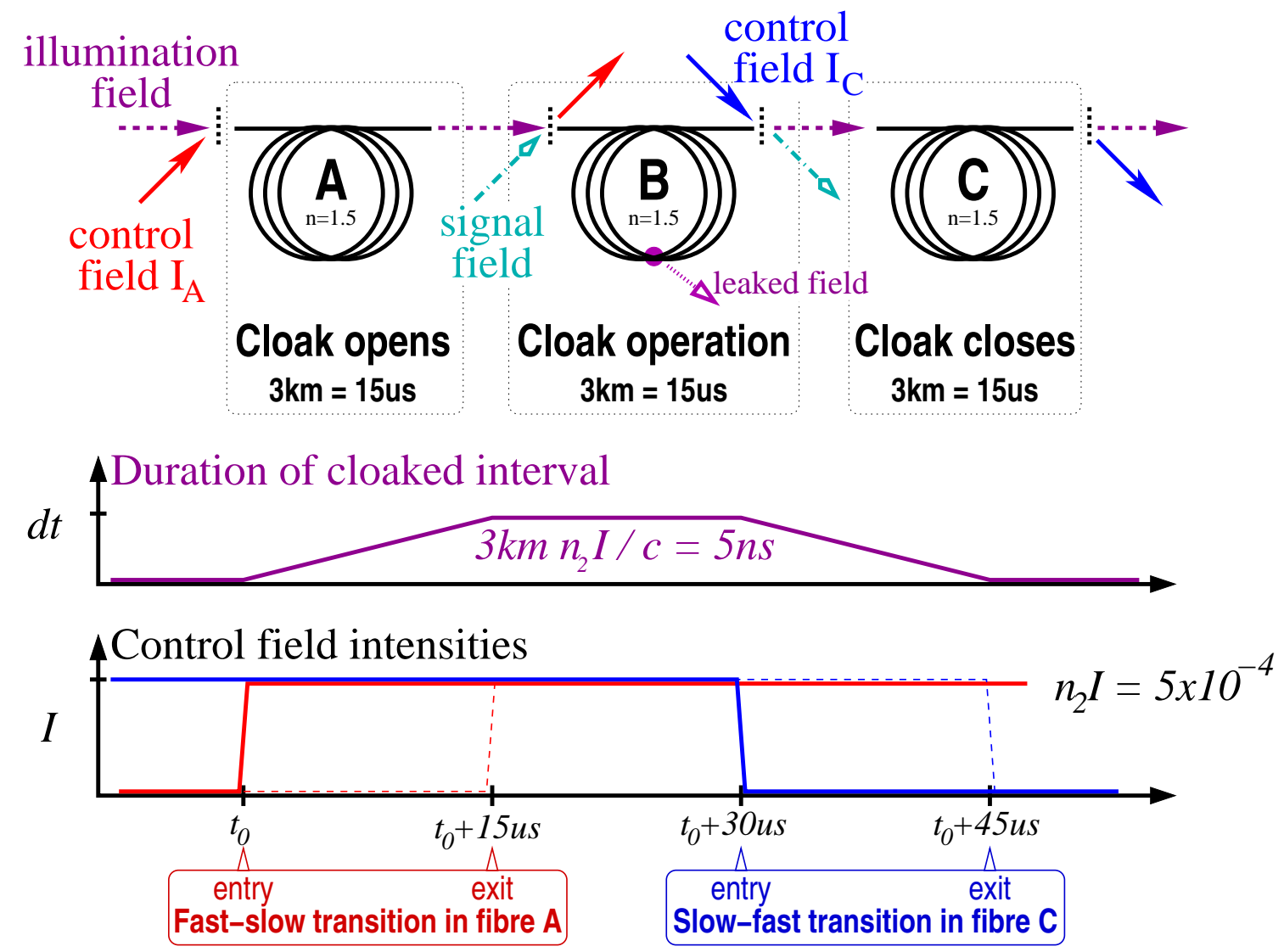

FIG. 5: Spacetime cloak based on exploiting the nonlinear refractive index properties of optical fibre. The cloak is opened in fibre A, is held open in fibre B (which contains the core cloak region), and is closed again in fibre C. Two control fields $I_{A}$ and $I_{C}$ modulate the refractive index in A (C) from low to high (high to low). The much weaker illumination field experiences the index changes required for the cloak illustrated in Fig. 3. A signal field injected directly into fibre B is present before, during, and after the cloaking from the illumination field. By monitoring the signal as it is coupled out, the spacetime modulation on it from the illumination and the effect of nonlinear refractive index can be measured. Changes to the signal field during the cloaking period leave no signature on the illumination field. Timing and synchronisation is only critical in ensuring that the top right apex of the cloaking diamond (Fig. 5) aligns with the bottom-left corner at an angle given by the average phase speed of the signals. Fibre parameters: silica refractive index $\sim 1.5$; control field intensity set to yield an index change $\Delta n=n_{2} I \sim 5 \times 10^{-4}$. 

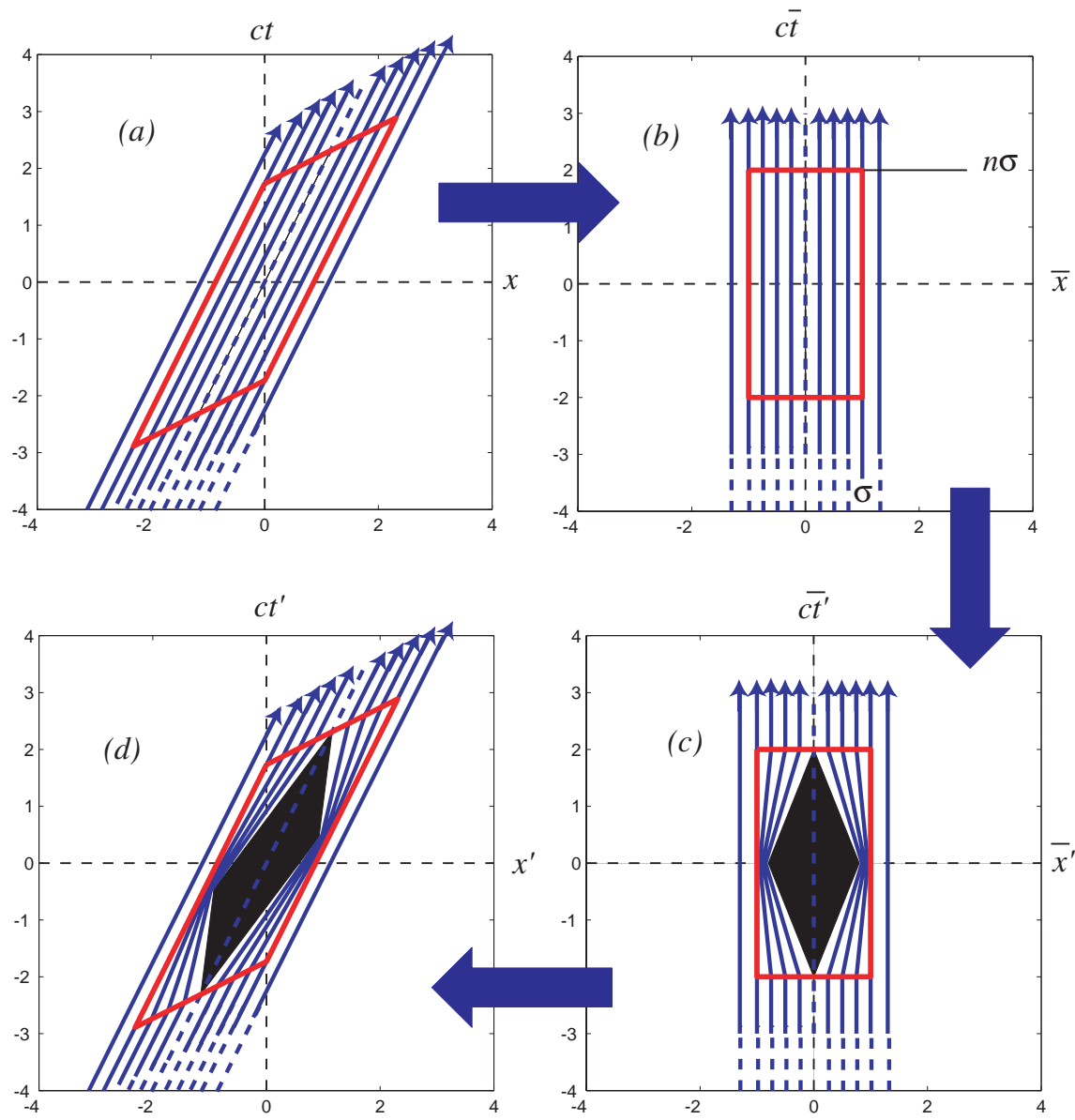

FIG. 6: The detailed construction of the curtain map 